\title{
Lidil
}

Revue de linguistique et de didactique des langues

40 | 2009

La motivation pour l'apprentissage d'une langue seconde

\section{Prolégomènes : où en est la recherche sur la motivation en LVE et en L2 ?}

Françoise Raby et Jean-Paul Narcy-Combes

\section{CpenEdition}

\section{Journals}

Édition électronique

URL : http://journals.openedition.org/lidil/2896

DOI : 10.4000/lidil.2896

ISSN : 1960-6052

Éditeur

UGA Éditions/Université Grenoble Alpes

Édition imprimée

Date de publication : 1 décembre 2009

Pagination : 5-16

ISBN : 978-2-84310-154-0

ISSN : $1146-6480$

Référence électronique

Françoise Raby et Jean-Paul Narcy-Combes, «Prolégomènes : où en est la recherche sur la

motivation en LVE et en L2 ? », Lidil [En ligne], 40 | 2009, mis en ligne le 01 juin 2011, consulté le 19 avril 2019. URL : http://journals.openedition.org/lidil/2896 ; DOI : 10.4000/lidil.2896

(c) Lidil 


\title{
Prolégomènes : où en est la recherche sur la motivation en LVE et en L2?
}

\author{
Françoise Raby* et Jean-Paul Narcy-Combes**
}

Les chercheurs en didactique des langues ont en commun de souhaiter œuvrer à une meilleure compréhension des processus d'enseignement/ apprentissage dans la classe de langue. Depuis quelques décennies, les progrès réalisés dans le domaine de la psychologie et de la sociologie, ainsi que dans celui des sciences du langage, ont permis à cette discipline encore jeune de progresser dans cette voie (Dörnyei, 2005, 2009), notamment grâce aux recherches sur la motivation en LVE ou en L2 ${ }^{1}$. Pendant longtemps, la question de la motivation en L2 est restée confinée dans le champ de la sociolinguistique, où les chercheurs stimulés par les travaux fondateurs de Gardner et Tremblay essayaient de comprendre ce qui gouvernait l'acquisition de la L2 parmi les migrants. Ces derniers analysèrent le cas des immigrés canadiens et démontrèrent que la maitrise de la L2 facilitait l'intégration et, qu'à l'inverse, le désir de s'intégrer dans une communauté allophone facilitait l'apprentissage de la L2. Parallèlement, un autre paradigme de recherche sur la motivation se développait dans le champ de la psychologie cognitive, qui donna naissance à pléthore de théories et de courants dont les plus aboutis furent la théorie socio-éducative (Gardner, 1985), la théorie

* IUFM, Univ. Joseph Fourier, LIDILEM, Université Stendhal - Grenoble 3.

** Université Paris 3.

1. Aujourd'hui, les chercheurs ont indistinctement recours à l'expression «langue seconde» pour désigner toute forme de langue étrangère. Ils ne font pas la distinction entre la langue apprise par des étrangers dans le pays d'adoption (langue endolingue), qui fait l'objet de toutes sortes de renforcement en dehors de l'école (langue vivante étrangère ou LVE), et la langue étrangère apprise dans son propre pays à l'école (langue exolingue), généralement qualifiée de langue seconde (L2). Il nous semble toutefois utile de conserver cette distinction dans les recherches sur la motivation, tant l'effet du contexte y est important. 
de l'autodétermination (Deci et Ryan, 1985, 2002) et le modèle hiérarchique de Vallerand (1997), la théorie des buts d'accomplissement (Dweck, 1986, 1989; Maehr et Braskamp, 1986; Nicholson, 1984), la théorie de l'autorégulation (Bandura, 2003), la théorie de la motivation au travail (Francès, 1995) et la théorie de la motivation scolaire (Viau, 1999).

Plus tard, autour des années 1980, survint ce que d'aucuns ont considéré comme un changement de paradigme issu de la rencontre entre les psychologues intéressés par l'acquisition de la langue étrangère en situation académique et les didacticiens de langues intéressés par la psychologie de la motivation: the educational schift (le changement issu de l'éducation) (Dörney, 2001). Autrefois, la psychologie cognitive apparaissait, selon les mots de Defay (2005), comme une cognition «froide». Préoccupée uniquement de connaissances, de mémoires, de savoirs, de tâches, de performances et d'évaluation, elle laissait à d'autres le soin de se pencher sur les émotions, les affects, la cognition «chaude». Aujourd'hui, l'ancienne opposition a disparu et les chercheurs qui travaillent sur la motivation en psychologie cognitive tentent non seulement de comprendre la façon dont les connaissances se construisent, mais également le rôle que jouent nos croyances et nos affects dans nos relations au savoir et aux autres et, surtout, les interactions entre ces facteurs. Les didacticiens de langues, de leur côté, ne s'intéressent pas seulement au comment de l'apprentissage de la langue mais aussi au pourquoi, et posent la question : qu'est-ce qui motive les apprenants à s'investir ou non dans l'apprentissage d'une langue et culture en situation académique (l'école, le lycée ou le collège, l'université, les centres de langues, les écoles de langues, etc.) ? Qu'est-ce qui freine ou anéantit leur désir d'apprendre et leurs efforts?

\section{Quelques réflexions liminaires sur l'épineuse question de la motivation}

Les questions posées à la recherche sur la motivation sont, somme toute, assez simples :

1. Qu'est ce que la motivation? La question ontologique.

2. Comment sait-on que quelqu'un est motivé? Les indicateurs.

3. Quels sont les facteurs qui agissent sur la motivation et comment? Le pourquoi. 
Mais une fois posées ces questions, on ne s'étonnera pas que les scientifiques aient tant de mal à définir un état motivationnel et que cet état puisse être décrit selon des formalismes très différents. Car la manière dont on aborde cette question dépend simplement, comme l'a très bien montré Nuttin, de la conception qu'on a du développement et du fonctionnement de tout être humain en interaction avec son milieu. Dans son livre Théorie de la motivation humaine (1980), Nuttin relate ce qu'ont été les péripéties de la motivation (p. 25-33) avant que le cognitivisme ne s'empare du sujet. La motivation fut définie comme stimulus et charge d'énergie par les psychanalystes comme Freud et les éthologistes (Lorenz, 1937), mais aussi comme une association apprise chez les behavioristes (Skinner, 1967), et chacune de ces définitions portait la marque de la discipline ou du champ scientifique dans lequel le chercheur évoluait. À la manière de l'esprit chez Sternberg (1990), la motivation ressemblait finalement plus à une métaphore qu'à un concept.

L'accord est loin d'être réalisé entre psychologues au sujet de la place qu'il convient de réserver à la motivation dans l'étude et l'explication du comportement. Considérée par certains comme une notion floue destinée à disparaitre du vocabulaire de la psychologie expérimentale, la motivation se présente à d'autres comme le thème principal de la psychologie et la clé même de la compréhension de la conduite. On constate à la base de ce désaccord une diversité de points de vue qui font de la motivation une notion très confuse. (Nuttin, 1980, p. 25)

Si la confusion demeure aujourd'hui, le paradigme socioconstructiviste dominant a permis de mettre d'accord les théoriciens sur bien des points : la motivation ne se confond pas avec le désir ou l'intérêt, elle suppose aussi le passage à l'action et le maintien de l'effort; elle est le produit de facteurs cognitifs, affectifs et sociaux; c'est un état instable qui fluctue en fonction de l'expérience du sujet; enfin, la motivation est un construit du chercheur, elle ne s'observe pas directement (Narcy-Combes, Narcy-Combes et Starkey-Perret, ce dossier; Raby, 2008, 2009).

Au-delà de ces points de consensus, pour «expliquer» la motivation, nous nous trouvons devant une inflation de concepts et de modèles qui constituent, en fait, autant d'arpèges sur le thème des relations entre l'individu et son milieu. Ces variations proviennent du fait que les chercheurs, tout au long du vingtième siècle et un peu partout dans le monde, ont développé leurs propres modèles et théories, sans toujours connaitre le travail mené par d'autres sur d'autres continents. 
Ainsi, les théories qui apparaitront au fil des pages de ce dossier ne se contredisent pas nécessairement. La théorie du concept de soi (Markus et Nurius, 1986; Schunk, 1987, 1989) ne s'oppose pas à la théorie des buts d'accomplissement (Dweck, 1989); la théorie de l'attribution de Weiner (1992) ne s'oppose pas à celle du sentiment d'efficacité perçue par (c'est la théorie qui est perçue, ou le sentiment?) Bandura (2003), ni à celle de l'autodétermination de Deci et Ryan (1985). C'est d'ailleurs pour cette raison qu'on a bien souvent l'impression que, derrière une terminologie différente, les discours se recoupent. Malgré tout, des différences subsistent dans les angles d'approche de la motivation, dans l'ampleur des modèles et dans la manière dont on prétend mesurer l'état motivationnel. C'est ce qui fait la richesse du domaine mais aussi sa fragilité, il faut bien en convenir. Comme les travaux sur la motivation en L2 ou en LVE sont issus de ces recherches, on y trouve la même diversité et parfois, les mêmes sources de confusion.

À l'heure actuelle, le courant dominant les recherches sur la motivation est caractérisé par l'approche cognitive qui met l'accent sur les connaissances, les pensées, les réflexions des individus et leur manière d'interpréter l'expérience.

Humans are social animals, but they are also noteworthy for being self-aware. Indeed, the capacity for self-reflection, and especially for self-concepts formation, distinguishes humans from virtually every other species. Research on this unique feature of human psychology has demonstrated that the self is more than an epiphenomenon, but rather functions as an important platform for the regulation of thought, emotion, and interpersonal relations. (Nowak, Vallacher et Zochowski, 2005, p. 368)

Cette approche constitue une rupture avec l'approche béhavioriste, qui voyait les choses en termes de stimuli et de renforcements, ou avec les approches psychanalytiques qui parlaient d'instincts, de pulsions (drive), d'états émotionnels. Cependant, aucun chercheur, actuellement, ne pourrait nier le rôle des émotions dans le déclenchement ou le maintien de la motivation : colère, anxiété, attente, fierté, etc. Ainsi, Raby (ce dossier), qui inscrit sa réflexion sur la motivation dans une perspective ergonomique, met-elle à jour l'impact du champ de contrôle de l'action sur les affects et les émotions d'enseignants d'anglais LVE au cours de l'action.

C'est pourquoi, alors que les deux aspects étaient traités séparément, on tente aujourd'hui de trouver leur articulation. C'est le cas notam- 
ment de la théorie des attributions de Weiner (1992). En ce qui concerne l'apprentissage des langues étrangères, Schumann, dans son livre The Neurobiology of Affect in Language (1998), affirme qu'il s'agit d'un processus fondamentalement piloté par l'émotion, et Arnold (1999) et Young (1999) lui font écho. Enfin, la dimension sociale, surdéterminée de la L2 ou de la LVE, ne fait plus de doute, comme en témoignent, dans ce volume, les articles de Narcy-Combes, Narcy-Combes et StarkeyPerret ainsi que de Serge Dreyer.

Dans les pays anglo-saxons, la motivation pour la LVE en SLA constitue, depuis des décennies, un champ de recherche important, mais surtout depuis les travaux du Canadien Robert Gardner (pour une revue de la question, voir Dörnyei, 2001, 2003, 2009; Oxford, 1999). Dans la littérature francophone, en revanche, son développement est beaucoup plus récent (Prince, 2008; Ishikawa, 2006; Lantolf et Genung, 2000; Raby, 2007, 2008). Il a été encouragé en psychologie par les travaux fondateurs de Nuttin (1980) et s'est parallèlement développé en sciences de l'éducation (Vallerand et Miquelon, 2008) grâce, notamment, à l'apport de la psychologie sociale et au renouvellement des approches sociologiques dans ce domaine. Il semblait inévitable que la didactique des langues ne reste pas étrangère à ces évolutions, car la question de la motivation se posait, de manière quotidienne et cruciale, en classe de langue. Or, si dans la littérature francophone, la motivation pour la LVE à l'école est un domaine scientifique encore relativement jeune, elle semble en pleine expansion : en 2004, 1'Association des professeurs de langues des IUT (APLIUT) lui a consacré son congrès de $2004^{2}$ et Les Lāngũes Modernes, ũn nũmpro sppciāl en

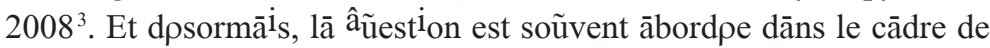

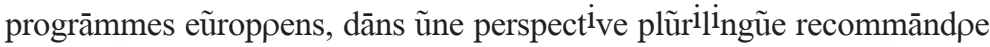
pār le Conseil de l'Eũrope en $2007^{4}$.

Les sept articles qui constituent ce numéro tentent de fournir des éléments de réponse à cette question. Certains adoptent une approche orientée plutôt «contenu», centrée sur les concepts, d'autres se situent

2. Les Cahiers de l'APLIUT, vol. XXIV, $\mathrm{n}^{\circ} 2$, juin 2005, éditorial.

3. Les Langues Modernes, $\mathrm{n}^{\circ}$ 3, 2008, coordonné par F. Raby.

4. De la diversité linguistique à l'éducation plurilingue : Guide pour l'élaboration des politiques éducatives en Europe [en ligne], Strasbourg, Conseil de l'Europe (2007), disponible sur <http://www.coe.int/t/dg4/Linguistic/ Guide_niveau3_FR.asp> [consulté le 05/10/2009]. 
plutôt du côté des processus, des dispositifs. Il ne s'agit pas d'une opposition tranchée mais plus d'un continuum dans lequel chaque article pourrait s'insérer.

Les approches «contenu» accordent une place importante à la conceptualisation de la motivation : elles cherchent à identifier un agrégat de facteurs qui poussent les individus à agir et à comprendre les relations qui s'établissent entre leurs motivations et leurs actions. Dans cette approche, on considère chaque facteur comme un élément stable, susceptible d'être extrait à partir de batteries de questionnaires ou bien à l'aide d'entretiens. Parfois, les données sont croisées avec des performances, afin de savoir si les variables motivationnelles sont en corrélation avec les performances des apprenants en LVE ou langue seconde (voir Brown, ce dossier). Par exemple, dans une récente méta-analyse, les auteurs ont trouvé que la motivation était le meilleur "prédicteur» de succès, alors que les orientations ou les attitudes envers la situation d'apprentissage n'agissaient qu'indirectement sur les performances, c'est-à-dire via la motivation (Masgoret et Gardner, 2003).

Les approches dynamiques (de type processus) relèvent souvent d'une approche locale et située (Dörnyei, 2003, 2009). Elles visent à préciser comment les variables évoquées plus haut interagissent dans l'évolution du comportement des sujets. Elles constituent le courant dominant des recherches sur la motivation pour la LVE en situation académique, car l'étude des situations de formation exige que la motivation soit envisagée sur le plan temporel, de manière dynamique, afin de repérer en quoi le contexte ou les dispositifs d'apprentissage affectent son évolution. Nous sommes donc passés d'une approche structuraliste, de type «état», à une approche plus dialectique, de type «processus » (Dörnyei, 2001; Julkunen, 2001).

\section{Présentation du dossier}

L'article de Christelle Goffin, Annick Fagnant et Christiane Blondin : Les langues des voisins : des langues très appréciées?, d'inspiration socioconstructiviste, introduit ce dossier. Il peut être considéré comme emblématique de l'approche par les contenus car il présente une enquête menée auprès de jeunes lycéens dans divers pays européens. Le but des auteurs est de savoir si la proximité d'un pays s'accompagne d'une attitude positive vis-à-vis de sa communauté et de la langue qu'on y parle. Une telle attitude se traduirait alors par une motivation intégrative vis-à-vis de cette langue que l'élève choisirait comme L2 à l'école. 
L'enquête montre qu'il n'en est rien et que d'autres facteurs plus puissants viennent contrecarrer l'influence de la proximité.

L'article de Serge Dreyer, Apprentissage du français et motivation existentielle. Le cas des universités à Taiwan, se situe clairement dans le contexte de la L2. Il s'inscrit dans la même problématique que celui de Fumiya Ishikawa, Impact des motivations sur la faculté de langage en interaction didactique : comment motiver les apprenants asiatiques à apprendre le français langue étrangère? Mais leur approche est différente. Serge Dreyer adopte ici une approche de type «contenu», fondée sur une analyse sociologique des représentations sociales du français langue étrangère chez les Taiwanais et de leur impact sur la motivation des étudiants. En forgeant le concept de motivation existentielle, il nous permet de comprendre comment l'apprentissage du français langue étrangère contribue à la formation identitaire (Dörnyei, 2009) des étudiants taiwanais et devient source de motivation.

Fumiya Ishikawa, de son côté, part du même constat pour analyser l'impact d'un dispositif didactique sur cette double motivation centripète (le désir de culture française) et centrifuge (la confrontation à l'étranger qui conduit à l'affirmation de soi). Les observations qu'il a menées en classe montrent comment ce double mouvement agit positivement sur le processus d'acquisition de la L2 par les étudiants.

C'est bien de dispositif et de processus qu'il est également question dans les articles de Peter Prince, Un ménage à trois difficile; Autonomie, Motivation et Apprentissage dans un Centre de Langues et de Lydie Lozinguez-Ben Gayed et Annick Rivens Mompean, L'impact d'une cyberquête sur la motivation d'apprenants de spécialité. Mais là encore, les approches diffèrent. Peter Prince a réalisé une enquête de type macropsychologique auprès d'étudiants d'un centre de langues en auto-apprentissage. Cette enquête indique que l'autonomie n'est pas forcément un facteur de motivation, cette dernière dépendant de la combinaison de plusieurs facteurs dont l'autonomie, mais aussi le niveau de langue et/ou l'image de soi.

Lydie Lozinguez-Ben Gayed et Annick Rivens Mompean, de leur côté, livrent une analyse clairement située du côté des processus, qui s'inscrit dans l'approche de la motivation par les tâches et les dispositifs (Julkunen, 2001; Raby, 2007). Elles ont mis en œuvre un dispositif de type cyberquête (Mangenot et Louveau, 2006) pour stimuler la motivation de leurs étudiants en langue de spécialité. Elles montrent l'impact positif d'un dispositif médiatisé sur la motivation et l'expliquent par deux facteurs : la baisse du sentiment d'appréhension vis-à-vis de 
la tâche en LVE et le sentiment d'avoir pu construire des compétences transversales, comme une meilleure utilisation de l'Internet.

David Brown se range également du côté des processus. Son expérience d'enseignement de l'anglais dans des écoles d'ingénieur ou d'architecte en France le conduit à poser la question suivante : les théories anglo-saxonnes de la motivation sont-elles pertinentes pour expliquer la motivation à apprendre une langue dans toutes les cultures? Il prend l'exemple du modèle de l'autodétermination de Deci et Ryan (2002) qui postule (tout en admettant qu'il s'agit en réalité d'un continuum) qu'un étudiant orienté intrinsèquement est nécessairement plus efficace qu'un étudiant orienté extrinsèquement. La recherche qu'il a mise en place auprès d'étudiants français ingénieurs et architectes montre que ce n'est pas le cas pour les étudiants français habitués à travailler dans un but de performance plus que de maitrise.

Enfin, le dossier conclut en abordant la question de la motivation des enseignants, un thème que nous avons tous deux retenus sans nous consulter. Françoise Raby, La dissonance motivationnelle ou l'impact d'un environnement hautement dynamique sur la motivation des enseignants de langues, adopte une approche type processus. À l'instar de Lozinguez-Ben Gayed et Rivens Mompean, elle étudie l'impact d'un dispositif de type cyberquête mais, cette fois-ci, sur la motivation des enseignants qui mettent en œuvre le dispositif. Inscrite dans la perspective de la psychologie ergonomique (Hoc, 1996), l'analyse des entretiens menés auprès de trois enseignants d'anglais montre que la diminution du champ de contrôle de l'enseignant dans ces environnements dynamiques que sont les classes multimédia, menace la motivation en créant un état de dissonance motivationnelle.

Pour leur part, Jean-Paul Narcy-Combes, Marie-Françoise NarcyCombes et Rebecca Starkey-Perret adoptent, dans Discours des enseignants sur leur formation et leur métier : quel lien avec la motivation des élèves?, une approche sociologique pour s'interroger sur la motivation des enseignants de langue dans la France d'aujourd'hui. C'est une approche de type «contenu» qui fait appel au concept d'investissement élaboré par Norton (1995) à la suite de Bourdieu et Passeron (1970). On peut considérer ce concept comme le pendant, sur le plan social, de l'implication sur le plan psychologique. Le deuxième concept invoqué pour expliquer la motivation est celui de retour sur investissement par rapport à l'investissement consenti. L'enquête qu'ils ont menée auprès d'enseignants de LVE en France révèle que ces derniers ne sont globalement satisfaits ni du retour matériel (salaires, organisation du travail, 
disponibilité) ni du retour symbolique (sentiments de reconnaissance sociale de la valeur de leur mission et de leur compétence). Ils posent alors, pour conclure, le risque de conséquences négatives de ce retour insuffisant du point de vue de la motivation des enseignants à s'investir dans leur métier et, par ricochet, du point de vue de la motivation des élèves eux-mêmes.

\section{Conclusion}

De nouvelles perspectives de recherches apparaissent : comment prendre en compte la diversité des facteurs et surtout leurs interactions, ce qui constitue sans doute l'enjeu majeur des recherches à venir? Quel type de méthodologie hybride ou mixte peut nous permettre de progresser sur cette voie? La triangulation des sujets, des données, des théories est-elle une voie prometteuse? Quelle place donner à la LVE en tant qu'objet d'apprentissage, objet de pratique, objet de culture, dans l'élaboration du construit motivationnel? Quels liens établir entre la motivation pour la L2 et la motivation pour la LVE, et entre la motivation pour la L2 et la L1? Autant d'interrogations, autant de défis à relever dans l'avenir pour les chercheurs en motivation en didactique des langues et en acquisition de la langue seconde.

\section{RÉFÉRENCES BIBLIOGRAPHIQUES}

ARnold J. (éd.) (1999) : Affective Language Learning, New York, Cambrige University Press.

BANDURA A. (2003) : Auto-efficacité : le sentiment d'efficacité personnelle, Bruxelles, De Boeck.

Bourdieu P. et PASSERON J. C. (1970) : La reproduction, Paris, Les Éditions de Minuit.

Deci E. L. et Ryan R. M. (1985) : Intrinsic motivation and self-determination in human behavior, New York, Plenum.

DeCI E. L. et RyAN R. M. (2002) : Handbook of self-determination research, Rochester, The University of Rochester Press.

Defays J.-M. (2005) : «De l'enseignement à l'apprentissage, le paradigme cognitif en didactique des langues », Revue Parole, $\mathrm{n}^{\circ}$ 34-35-36, p. $165-202$.

DÖRNYEI Z. (2001) : Teaching and researching motivation, Londres, Éd. Longman. 
Dörnyei Z. (2003) : «Attitudes, Orientations and Motivations in Language Learning, Advances in Theory, Research, and Applications », Language Learning, vol. 53, n 1, p. 3-32.

DöRNYEI Z. (2005) : The psychology of the language learner, Mahwah, Lawrence Erlbaum Associates.

DöRNYEI Z. (2009) : The psychology of second language acquisition, Oxford, Oxford University Press.

DöRnyei Z. et Ushioda E. (2009) : Motivation, language identity and the L2 self, Bristol, Bristol Multibilingual papers.

Dweck C. S. (1986) : «Motivational processes affecting motivation », American Psychologist, vol. 41, p. 1040-1048.

Dweck C. S. (1989) : «Motivation», dans A. Lesgold et R. Glaser (éds), Foundations for a psychology of education, Hillsdale, New Jersey Hove, Lawrence Erlbaum Associates, p. 87-106.

GARDNER R. C. (1985) : The Attitude Motivation Test Battery: Technical Report, Unpublished Report, University of Western Ontario, London, Ontario, Canada.

Hoc J. M. (1996) : Supervision et contrôle de processus. La cognition en situation dynamique, Grenoble, Presses Universitaires de Grenoble.

IshiKawA F. (2006) : «L'enseignement du français au Japon : une didactique du FLE mise en jeu par la réalité extérieure de la classe», dans $\mathrm{V}$. Castelloti et $\mathrm{H}$. Chalabi (éds), Le français langue étrangère et seconde : des paysages didactiques en contexte, Paris, L'Harmattan, p. 133-143.

JULKUNEN K. (2001) : «Situation and task-specific motivation in second language learning and teaching», in Z. Dörnyei et Schmidt R. (éds), Motivation and second language learning, Honolulu, University of Hawaii Press, p. 29-42.

Lantolf J. P. et Genung P. B. (2000) : L'acquisition scolaire d'une langue étrangère vue dans la perspective de la théorie de l'activité : une étude de cas [en ligne], mis en ligne le 16 décembre 2005, disponible sur $<$ http://aile.revues.org/document280.html> [consulté le 05/10/2009].

Lorenz K. (1937) : «Ueber den Begriff der Instintkthandlung», Folia Biotheoretica, 2, p. 18-50.

Maehr M. L. et Braskamp L. A. (1986) : The motivational factor: A theory of personal investment, Lexington, Lexington books.

Mangenot F. et Louveau É. (2006) : Internet et la classe de langues, Paris, CLE international.

Markus H. et Nurius P. (1986) : «Possible selves», American Psychologists, vol. 41, p. 954-969. 
Masgoret A. M. et Gardner C. (2003) : «Attitudes, Motivation and Second Language Learning: a Meta Analysis of Studies Conducted by Gardner and Associates », Language Learning, vol. 53, $\mathrm{n}^{\circ}$ 1, p. 167-210.

Nicholson J. G. (1984) : «Conception of Ability and Achievement Motivation», dans R. E. Ames, et C. Ames (éds), Research on Motivation in Education, vol. 1, Toronto, Toronto Academic Press, p. 39-73.

Noels K. A., Pelletier L. G., Clément R. et Vallerand R. J. (2003) : «Why are you learning a second language, Motivational, Orientations and Self-Determination Theory », Language Learning, vol. 53, $\mathrm{n}^{\circ} 1$, p. 33-63.

Norton P. (1995) : «Social identity, investment, and language learning», TESOL Quarterly, vol. 29, n 1, p. 9-31.

Nowak A. et Vallacher R., et Zochowski M. (2005) : «The emergence of personality: dynamic foundations for individual variations », Developmental review, 25, p. 351-385.

Nuttin J. (1980) : Théorie de la motivation humaine, Paris, PUF.

Prince P. (2008) : «La motivation dans un centre de langue ou le pari de l'autonomie», Les langues modernes, $\mathrm{n}^{\circ} 3$, p. 37-43.

RABY F. (2007) : «A triangular approach to motivation in Computer Assisted Autonomous Language Learning (CAALL)», Recall, vol. 19, n 2, p. 181-201.

RABY F. (2008) : «Comprendre la motivation en LV2 : quelques repères venus d'ici et d'ailleurs », Les langues modernes, n 3, p. 9-16.

RABY F. (2009) : «La motivation pour l'apprentissage d'une langue vivante en débat», La motivation dans l'enseignement des langues : comment promouvoir le gout du français en Asie de l'Est, (actes du colloque de la Société coréenne d'enseignement de langue et de littérature françaises, Université féminine de Sookmyung, 10 octobre 2009).

Schumann J. H. (1998) : The neurobiology of affect in language, Oxford, Blackwell.

Schunk D. H. (1987) : «Self-efficacy and motivating learning», dans N. Hasting et J. Schwieso (éds), New directions in educational psychology, Londres, Falmer Press, 2, p. 233-251.

Schunk D. H. (1989) : «Self-efficacy an Cognitive Achievements. Implications for Students with Learning Problems », Journal of learning disabilities, vol. 22, $\mathrm{n}^{\circ} 1$, p. 14-22.

Skinner B. F. (1967) : Science and Human Behavior, New York, New York Free Press.

Stenberg R. (1990) : Metaphors of mind, Cambridge, Cambridge University Press. 
VALLERAND R. J. (1997) : «Toward a Hierarchical model of intrinsic and extrinsic motivation», dans M. R. Zanna (éd.), Advances in experimental social psychology, 29, New York, Academic Press, p. 271-360.

Vallerand R. J. et Miquelon P. (2008) : «Le Modèle Hiérarchique : une analyse intégrative des déterminants et conséquences de la motivation intrinsèque et extrinsèque », dans R. Joule (éd.), Bilans et perspectives en psychologie sociale, Grenoble, Presses Universitaires de Grenoble.

VIAU R. (1999) : La motivation en contexte scolaire, Bruxelles, De Boeck.

WeINER B. (1992) : Human motivation: Metaphors, theories and research, Newbury Park, Sage.

Young D. J. (éd.) (1999) : Affect in foreign language and second language learning, Boston, McGraw-Hill. 